\title{
On the representation of signals series by Faber-Schauder system
}

\author{
Tigran Grigoryan ${ }^{1}$, and Martin Grigoryan ${ }^{2}$ \\ ${ }^{1}$ Yerevan State University, Chair of Higher Mathematics, , 1 Al. Manukyan str., Yerevan, 0025, Armenia \\ ${ }^{2}$ Yerevan State University, Chair of Higher Mathematics, , 1 Al. Manukyan str., Yerevan, 0025, Armenia
}

\begin{abstract}
We prove that: if $f(\mathrm{x})$ is a measurable function, finite almost everywhere on $[0,1]$ then there is a Schauder series, which converges unconditionally to $f$ almost everywhere on $[0,1]$ and for every $\varepsilon>0$ there is a measurable set $E$ contained in $[0,1]$, with $|E|>1-\varepsilon$, such that for each $f \in C(E)$ there is a Schauder series, which unconditionally converges to $f$ in the uniform norm.
\end{abstract}

\section{Introduction}

First, we recall the definition of the Faber-Schauder system (see [2]):

$$
\left\{\varphi_{n}(x): n=0,1, \ldots\right\}, x \in[0,1]
$$

where

$$
\varphi_{0}(x)=1 \text {, }
$$

$\varphi_{1}(x)=x$ and for $n=2^{k}+i ; k=0,1 \ldots ; i=1,2, \ldots, 2^{k}$ :

$$
\varphi_{n}(\mathrm{x})=\varphi_{k}^{(\mathrm{i})}(\mathrm{x})=\left\{\begin{array}{l}
\text { if } x \notin\left(\frac{i-1}{2^{k}}, \frac{i}{2^{k}}\right), \\
1 \text { if } x=x_{n}=x_{k}^{(\mathrm{i})}=\frac{2 i-1}{2^{k+1}}, \\
\text { linear and continuous on }\left[\frac{i-1}{2^{k}}, \frac{2 i-1}{2^{k+1}}\right],\left[\frac{2 i-1}{2^{k+1}}, \frac{i}{2^{k}}\right] .
\end{array}\right.
$$

For $2 \leq n=2^{k}+i$ we set $\Delta_{n}=\Delta_{k}^{(i)}=\left(\frac{i-1}{2^{k}}, \frac{i}{2^{k}}\right)$, the set of points, where the function $\varphi_{n}(x)$ is different from zero.

We also recall that the Faber-Schauder system forms a basis in the space $C[0,1]$ (see [2]) that is, any function $f \in C[0,1]$ can be uniquely represented by the series Fourier series

$$
f(x)=\sum_{n=0}^{\infty} A_{n}(f) \varphi_{n}(x)
$$

with respect to the Faber-Schauder system, which converges to $f$ uniformly on $[0,1]$. The $A_{n}(f)$ coefficients of this series are defined by $A_{0}(f)=f(0), A_{1}(f)=f(1)-f(0)$,

$A_{n}(f)=A_{k, i}(f)=f\left(\frac{2 i-1}{2^{k+1}}\right)-\frac{1}{2}\left[f\left(\frac{i-1}{2^{k}}\right)+f\left(\frac{i}{2^{k}}\right)\right]$.

There are number of interesting results on the properties of the Faber-Schauder system (see [2-9]).

Definition 1. A basis $\left\{f_{k}: k=1,2 \ldots\right\}$ of a Banach space $B$ is said to be unconditional if for an arbitrary permutation $\left\{\sigma_{k}: k=1,2 \ldots\right\}$ of the natural numbers, the system $\left\{f_{\sigma(k)}(x): k=1,2 \ldots\right\}$ is also a basis of $B$.

Let $E \subset[0,1]$ be a measurable set and let $C(\mathrm{E})$ be the class of all continuous functions defined on $E$, and let $b_{n}(x) \in C(E), n=1,2 \ldots$.

Definition 2. The series $\sum_{n=1}^{\infty} b_{n}(x)$ is said to be unconditionally convergent to $f(\mathrm{x})$ in $C(\mathrm{E})$ if for any permutation $\{\sigma(k): k=1,2 \ldots\}$ of the natural numbers, the series $\sum_{n=1}^{\infty} b_{\sigma(k)}(x)$ converges to $f(\mathrm{x})$ uniformly on $E$.

Definition 3. The system $\left\{f_{k}: k=1,2 \ldots\right\}$ is said to be an unconditional system of the representation of functions in the class $C(\mathrm{E})$ if for every function 
$f(\mathrm{x}) \in C(\mathrm{E})$ there exists a series $\sum_{n=1}^{\infty} a_{n} f_{n}(x)$ such that it unconditionally converges to $f(\mathrm{x})$ in $C(\mathrm{E})$.

We note that in the space $C[0,1]$ there exists no unconditional basis (see [10]).

And what is more, in the article [3] M. G. Grigorian and A. A. Sargsyan, constructed a continuous function $f_{0}(x) \in C[0,1]$ and a permutation $\{\sigma(k): k=1,2 \ldots\}$ of natural numbers, such that the series $\sum_{k=0}^{\infty} A_{\sigma(k)}\left(f_{0}\right) \varphi_{\sigma(k)}(x)$ diverges in measure on [0,1] and $\quad\left|A_{\sigma(k)}\left(f_{0}\right)\right|<\left|A_{\sigma(k-1)}\left(f_{0}\right)\right|, \mathrm{k}>1$ (where $\sum_{n=0}^{\infty} A_{n}\left(f_{0}\right) \varphi_{n}(x)$-is $\quad$ Fourier series of function $f_{0}(x) \in C[0,1]$ with respect to the Faber-Schauder system).

From this follows: there exists a function $f_{0}(x) \in C[0,1]$, whose greedy algorithm with respect to the Faber-Shauder system diverges in measure.

But, nevertheless, for every $0<\varepsilon<1$ there exists a measurable set $E \in[0,1]$ with measure $|E|>1-\varepsilon$, such that for each $f \in C(E)$ one can find a function $g \in C[0,1], g(x)=f(x), \forall x \in E \quad$ equal to $f$ on $\mathrm{E}$ such that the greedy algorithm with respect to the FaberShauder system of signal $g$ converges it in the $C[0,1]$ - norm.

Now we recall the definition of the greedy algorithm.

Let $\Psi=\left\{\psi_{k}\right\}_{k=1}^{\infty}$ be a normalized basis in Banach space $\sigma \in D(\mathrm{f}, \psi)$. Then for each element $f \in X$ there exists a unique series by system $\Psi=\left\{\psi_{k}\right\}_{k=1}^{\infty}$ converging to $f$ in the norm of space $X$ :

$$
f=\sum_{k=0}^{\infty} c_{k}(\mathrm{f}) \psi_{k}
$$

Let an element $f \in X$ be given. We call a permutation $\sigma=\{\sigma(k): k=1,2 .$.$\} \quad of nonnegative$ integers decreasing and write $\sigma \in D(f, \psi)$, if

$$
\left|c_{\sigma(k)}(f)\right|<\left|c_{\sigma(k-1)}(f)\right|, k>1 .
$$

In the case of strict inequalities here $D(\mathrm{f}, \psi)$ consists of only one permutation.

We define the m-th greedy approximant of $f$ with regard to the basis $\Psi$ corresponding to a permutation by formula

$$
G_{m}(\mathrm{f}, \zeta)=\sum_{k=1}^{m} c_{\sigma(\mathrm{k})}(\mathrm{f}) \psi_{\sigma(\mathrm{k})}
$$

This nonlinear method of approximation was considered in the article [10] and is known as greedy algorithm.

We say that the greedy approximant of element $\mathrm{f}$ by system $\Psi$ converges, if for some $\sigma \in D(\mathrm{f}, \psi)$ we have

$$
\lim _{m \rightarrow \infty}\left\|G_{m}(\mathrm{f}, \psi)=f\right\|_{X}=0 .
$$

Greedy algorithms in Banach spaces with respect to normalized bases have been investigated by Temlyakov, DeVore, Konyagin, Wojtaszczyk, Korner and other authors (see [11]-[16]).

Note that $G_{m}(\mathrm{f}, \psi)=\sum_{k=1}^{m} c_{\sigma(\mathrm{k})}(\mathrm{f}) \psi_{\sigma(\mathrm{k})}$ gives the best m-term approximation in $L^{2}[0,1]$ - norm.

We present some results which have an immediate bearing on our investigations.

In the article [12] T. W. Korner answering a question raised by Carleson and Coifman constructed a continuous function, whose greedy algorithm with respect to the trigonometric systems diverges almost everywhere.

In the article [13] $\mathrm{V}$. N. Temlyakov constructed a function $\mathrm{f}$ that belongs to any $L^{p}, p>0$ (respectively $\mathrm{p}>2$ ), whose greedy algorithm with respect to the trigonometric system diverges in measure (respectively in $\left.L^{p}[0,1], p>2\right)$.

In the article [3] M. G. Grigorian and A. A. Sargsyan, constructed a continuous function $f \in \mathrm{C}[0,1]$, whose greedy algorithm with respect to the Faber-Shauder system diverges in measure.

The following question arises here.

Question 1. Does there exist a set $e$ of arbitrarily small measure such that, after modifying the values of an arbitrary function in $f \in L^{p}[0,1], p>2, p>1$, on $e$, the greedy algorithms for the modified function with respect to the Walsh and trigonometric systems converge to it (almost everywhere, in the norm of $L^{p}[0,1]$ uniformly)?

Note that the answer to Question 1 on the one hand depends on the prescribed system and the type of convergence, and on the other hand depends on whether the exceptional set on which the modification is performed depends on the function $\mathrm{f}(\mathrm{x})$ to be corrected or is universal.

We shall consider two versions of Question 1:

a) when the values of the function $f(\mathrm{x})$ are modified on a set of arbitrarily smallmeasure depending on the function;

b) when the exceptional set e on which the modification is performed is independent of the modified function $f(\mathrm{x})$ (that is, is universal and serves for a whole function class). 
Note the following question, which arises when we investigate the convergence of the greedy algorithm for the new, corrected function e $f(x)$ and is also of independent interest. Can the values of any function $f(x)$ in $\left.L^{p}[0,1]\right], p>1$, be modified on a set of small measure so that all the nonzero elements in the sequence of the Fourier coefficients of the function thus obtained with respect to the classical systems (in particular, the Walsh and trigonometric systems) are arranged in decreasing order?

It is important to note that, as was shown in the article [15], M. G. Grigoryan, K. S. Kazaryan and F. Soria, there exist a complete orthonormal system $\Phi=\left\{f_{k}(x)\right\}_{k=1}^{\infty}$ and a function $f \in L^{p}[0,1], p>2$, such that if $\mathrm{g}(\mathrm{x})$ is any function in $L^{p}[0,1], p>2$ with measue $|\{x \in[0,1], g(x)=f(x)\}|>0 \quad$ then it's greedy algorithm with respect to the system $\Phi=\left\{f_{k}(x)\right\}_{k=1}^{\infty}$ diverges in $L^{p}[0,1], p>2$.

Note also that well-known classical theorems by $\mathrm{N}$. N. Luzin and D. E. Men'shov "on correction" of functions (see $[17,18]$ ). We have considered the behavior greedy algorithm after modification of functions (see [19-22]).

For Haar system we obtained:

For any $\varepsilon>0$ there exists a measurable set $E \subset[0,1]$, with measure $|E|>1-\varepsilon$ such that for any function $f(x) \in L^{1}[0,1]$ one can find a function $g(x) \in L^{1}[0,1]$ equal to $f(x)$ on $E$ such that its greedy algorithm with respect to the Haar system converges to $g(x)$ in the $L^{1}[0,1]$-norm.

In the paper [3] M. G. Grigorian and A. A. Sargsyan was obtained

Theorem 1. For any $\varepsilon>0$ there exists a measurable set $E \subset[0,1]$, with measure $|E|>1-\varepsilon$ such that for any function $f(x) \in C[0,1]$ one can find a function $g(\mathrm{x})$ $\in C[0,1]$ equal to $f(x)$ on $\mathrm{E}$ such that its greedy algorithm with respect to the Faber-Schauder system converges to $g(x)$ uniformly on $[0,1]$.

But, nevertheless, for every $0<\varepsilon<1$ there exists a measurable set $E \subset[0,1]$ with measure $|E|>1-\varepsilon$, such that the Faber-Schauder system is an unconditional system of representation of functions of the class $C(E)$ (see [4]). This result is a consequence of the more general Theorem 1(see [9]), which is stated as follows:

Theorem 2. For every $\varepsilon>0$ there is a measurable set $E$ contained in $[0,1]$, with $|E|>1-\varepsilon$, such that for each $f \in C(E)$ one can find a function $g \in C[0,1], g(\mathrm{x})=f(x), \forall x \in E \quad$ equal to $f$ on $E$ such that the Fourier series with respect to the Faber-
Schauder systemof signal $\mathrm{g}$ unconditionally converges it in the $C[0,1]$-norm and

$$
\sum_{n=0}^{m}\left|A_{n}(g)\right| \varphi_{n}(x)<2|g(x)|, \forall x \in[0,1], \forall m>0 .
$$

\section{Proof of theorems}

In this article we prove the following theorems.

\subsection{Results}

Theorem 3. Corresponding to each $f \in C[0,1]$ there is a Schauder series that converges to $f$ on a set of full measure, such that for every $\varepsilon>0$ there is a measurable set $E$ contained in $[0,1]$, with $|E|>1-\varepsilon$, on which the series converges unconditionally to $f$ in $C(\mathrm{E})$.

This theorem is a consequence of the more general Theorem 4, which is stated as follows:

Theorem 4. If $f(\mathrm{x})$ is a measurable function, finite almost everywhere on $[0,1]$ then there is a Schauder series, which converges unconditionally to $f$ almost everywhere on $[0,1]$.

Of course, these results can not be improved by replacing the set $E$ with $[0,1]$, since as Karlin has shown, there is no unconditional basis for $C[0,1]$ (see [2]).

\subsection{Proof of the Theorem 4}

Let $f(\mathrm{x})$ is a measurable and finite almost everywhere function on $[0,1]$ be given, and let $f_{1}$ be a binary step function such that

$$
\left|\left\{x \in[0,1],\left|f-f_{1}\right|>\frac{1}{4}\right\}\right|<\frac{1}{4} .
$$

By virtue of Lemma 3 of [4], there is a measurable set $E_{1} \subset[0,1]$ and a Schauder polynomial $Q_{1}(x)=\sum_{n=2}^{m_{1}} A_{n} \varphi_{n}(x),\left|A_{n}\right| \leq \frac{1}{2}$ such that $Q_{1}(x)=f_{1}(x)$ for all $x \in E_{1},\left|E_{1}\right|>1-\frac{1}{4}$, for each $B_{1} \subset\left\{2, \ldots m_{1}\right\}$,

$$
\left|\sum_{n \in B_{1}} A_{n} \varphi_{n}(x)\right| \leq\left|f_{1}(x)\right| \text { for all } x \in E_{1} .
$$

Let the binary step function $f_{2}$ satisfy

$$
\left|\left\{x \in[0,1],\left|\left(f-Q_{1}\right)-f_{2}\right|>\frac{1}{8}\right\}\right|<\frac{1}{8},
$$


and again applying lemma 3 of [4] we find the Schauder polynomial of the form $Q_{2}(x)=\sum_{n=m_{1}+1}^{m_{2}} A_{n} \varphi_{n}(x),\left|A_{n}\right| \leq \frac{1}{3}$, which coincides with $f_{2}$ on the measurable set $E_{2} \subset[0,1]$, where $\left|E_{2}\right|>1-\frac{1}{8}$ and for each $B_{2} \subset\left\{m_{1}+1, \ldots, m_{2}\right\}$ $\left|\sum_{n \in B_{2}} A_{n} \varphi_{n}\right| \leq\left|f_{2}(x)\right|$ for all $x \in E_{2}$, where $\left|A_{n}\right| \leq \frac{1}{2}$ for each $n \in\left(m_{1}, m_{2}\right]$.

Then

$\left|f(x)-\left(Q_{1}+Q_{2}\right)(x)\right|<\frac{1}{8}$ for all $x \in G_{2} \cap E_{2}$,

where

$$
G_{2}=\left\{x \in[0,1],\left|\left(f-Q_{1}\right)-f_{2}\right|<\frac{1}{8}\right\}
$$

From this we obtain

$$
\begin{aligned}
& \left|\sum_{n \in B_{2}} A_{n} \varphi_{n}(x)\right| \leq\left|f_{2}(x)\right| \leq\left|\left(f-Q_{1}\right)(x)-f_{2}(x)\right|+ \\
& +\left|\left(f-Q_{1}\right)(x)\right|<\frac{3}{8} ; \forall x \in G_{2} \cap E_{1} \cap E_{2} .
\end{aligned}
$$

Proceeding thus inductively, one determines a sequence $\left\{Q_{j}(x)\right\}_{j=1}^{\infty}$ of Schauder polynomials

$$
Q_{j}(x)=\sum_{n=m_{j-1}+1}^{m_{j}} A_{n} \varphi_{n}(x),\left|A_{n}\right| \leq \frac{1}{j+1} ; \forall n \in\left(m_{j-1} m_{j,}\right],
$$

and a sequences $\left\{E_{j}\right\}_{j=1}^{\infty}$ and $\left\{G_{j}\right\}_{j=1}^{\infty}$ are measurable subsets of $[0,1]$ such that

$$
\left|G_{j} \cap E_{j}\right|>1-\frac{1}{2^{j+1}} ; j=1,2 \ldots\left|f(x)-\sum_{j=1}^{n} Q_{j}(x)\right|<\frac{1}{2^{n+1}} ;
$$

$\forall x \in G_{n} \cap E_{n} ; n \geq 1$,

and for each $B_{j}$ contained in the set $\left\{m_{j-1}+1, \ldots, m_{j}\right\}$,

$$
\left|\sum_{n \in B_{j}} A_{n} \varphi_{n}(x)\right|<\frac{3}{2^{n+1}} \forall x \in G_{j} \cap E_{j-1} \cap E_{j} ; j \geq 1 .
$$

Let

$$
\left|\sum_{j=1}^{\infty} A_{n} \varphi_{n}\right|=\left|\sum_{j=1}^{\infty} \sum_{n=m_{j-1}+1}^{m_{j}} A_{n} \varphi_{n}\right| .
$$

We set $B=\bigcup_{n=1}^{\infty} \bigcap_{k=n}^{\infty}\left(\mathrm{E}_{k} \cap \mathrm{G}_{k}\right)$.
Let $x \in B$, then for some $n_{0} \in N$ we have $x \in G_{n} \cap E_{n}, \forall n>n_{0}$. Indeed if $\pi$ be a permutation of $N$, and if $N_{n}$ be chosen so that $\left\{\pi(k): 1 \leq k \leq N_{n}\right\} \supset\left\{i: 1 \leq i \leq m_{n}\right\} \forall n>n_{0}$ then for arbitrary $M>N_{n}$ and $x \in G_{n} \cap E_{n}, \forall n>n_{0}$ it is true:

$\left|f(x)-\sum_{k=1}^{M} A_{\pi(k)}(x)\right| \leq\left|f(x)-\sum_{j=n+1}^{m_{j}} Q_{j}(x)\right|+\sum_{j=n+1}^{\infty} \frac{3}{2^{j+1}}<\frac{1}{2^{n+1}}+$ $+\frac{3}{2^{n+1}}=\frac{1}{2^{n-1}}$.

I. e. the series $\sum_{i=1}^{\infty} A_{n} \varphi_{n}=\sum_{j=1}^{\infty} \sum_{n=m_{j-1}+1}^{m_{j}} A_{n} \varphi_{n}$ converges unconditionally to $f$ almost everywhere on $[0,1]$ and $A_{n} \rightarrow 0$ as $n \rightarrow \infty$.

\subsection{Remark.}

Repeating the argument in the proof theorem 3 we arrive at following Theorem

Theorem 5. There is a Schauder universal series of the form $\sum_{i=1}^{\infty} b_{n} \varphi_{n}(x),\left|b_{k}\right| \searrow 0$ with the following properties:

1. For each measurable function $f(\mathrm{x})$ one can find a sequence $\left\{\xi_{k}\right\}_{k=1}^{\infty}, \xi_{k}=0$, or $1, k=1,2, \ldots$, such that the series, $\sum_{n=1}^{\infty} \xi_{n} b_{n} \varphi_{n}(x)$ converges unconditionally to $f$ almost everywhere on $[0,1]$.

2. For every $\varepsilon>0$ there is a measurable set $E$ contained in $[0,1]$, with $|E|>1-\varepsilon$, such that for each $f \in C(E)$ one can find a function $g \in C[0,1], g(x)=f(x), \forall x \in E \quad$ equal to $f$ on $E$ such that the Fourier series with respect to the FaberSchauder system of signal $g$ unconditionally converges it in the $C[0,1]$-norm, and

$$
\begin{aligned}
& A_{n}(g)=b_{n}, \forall n \in \operatorname{spec}(g)=\left\{n \in \mathrm{N}, A_{n}(g) \neq 0\right\} \\
& \sum_{n=0}^{m}\left|A_{n}(g)\right| \varphi_{n}(x)<2|g(x)|, \forall x \in[0,1], \forall m>0 .
\end{aligned}
$$

\section{References}

1. G. Faber, Uber die ortogonalen functionen des Hern Haar, Iahrscber Deutsch, Math. Verein, BD.195, 104-112 (1910).

2. J. Schauder, Zur Theorie stetiger Abbildungen in Funktionalraumen, Math. Z., 26(1927), 47-65.

3. M. G. Grigorian, A. A. Sargsyan, Nonlinear approximation of continuous functions by the 
Faber-Schauder system, Mat. Sb.vol. 199:5, p 629653 (2008).

4. T. M. Grigoryan, On the unconditional convergence of series with respect to the Faber-Schauder system, Analysis Mathematica, 39, 179-188 (2013).

5. A. A. Talalyan, Representation of measurable functions by series, Usp. Mat. Nauk, 15, no. 5 Russian Math. Surveys, 1, (Russian) (1960).

6. Casper Goffman,"Remark on a problem of Lusin", Acta Math.,vol 111, p. 63--72S (1964).

7. V. G. Krotov "On the series by the Faber-Schauder system and by bases in C[0,1]", Mat. Zamet.,vol. 14, p. 185--195 (1973).

8. M. G. Grigoryan, V. G. Krotov, Luzin's Correction Theorem and the Coefficients of Fourier Expansions in the Faber-Schauder System, Mat. Zametki 93, no. 2, 172-178 (2013).

9. M. G. Grigorian and T. M. Grigoryan, On the absolute convergece Schauder series, Advances in Theoretical and Applied Mathematics,V. 9, n. 1 (2014),

10. Karlin, Bases in Banach spaces, Duke Math. J., 15, 971-985 (1948).

11. R. A. DeVore, V. N. Temlyakov, "Some remarks on greedy algorithms", Advances in Computational Math., 5, 173-187 (1996).

12. T. W. Korner, Decreasing rearranged Fourier series, J. Fourier Anal. Appl. 5, no. 1, 1-19 (1999).

13. S. V. Konyagin and V. N. Temlyakov, "A remark on Greedy approximation in Banach spaces", East Journal on Approximations, 5:1, 1-15 (1999).

14. P. Wojtaszczyk, "Greedy Algorithm for General Biorthogonal Systems", Journal of Approximation Theory, 107, 293-314 (2000).

15. M. G. Grigoryan, K. S. Kazaryan and F. Soria, "Mean convergence of orthogonal Fourier series of modified functions", Trans. Amer. Math. Soc. 352:8, 3777-3798 (2000).

16. M. G. Grigoryan and R. E. Zink, "Greedy approximation with respect to certain subsystems of the Walsh orthonormal system", Proc. Amer. Math. Soc. 134, 3495-3505 (2006).

17. N. N. Luzin, On the fundamental theorem of the integral calculus, Sb. Math. 28, 266-294 (in Russian) (1912).

18. D. E. Menchoff [D. E. Men`shov], Sur la convergence uniforme des series de Fourier, Sb. Math. 53, no. 11, 67-96 (1942).

19. M. G. Grigoryan, Modifications of functions, Fourier coefficients and nonlinear approximation, Sb. Math. 203, no. 3, 49-78 (2012).

20. M. G. Grigorian (Grigoryan), On the convergence of Fourier series in the metric of $\mathrm{L}^{1}$, Anal. Math. 17, no. 3, 211-237 (1991).

21. M. G. Grigorian, Uniform convergence of the greedy algorithm with respect to the Walsh system, Studia Math. 198, no. 2, 197-206 (2010).
22. M. G. Grigoryan, L. N. Galoyan, On the uniform convergence of negative order Cesaro means of Fourier series, J. Math. Anal. Appl. 434, no. 1, 554567 (2016).

23. M. G. Grigoryan, A. A. Sargsyan, On the universal functions for the class Lp[0,1], J. Funct. Anal. 270, 3111-3133 (2016). 